\title{
Riccati equation, Factorization Method and Shape Invariance
}

\author{
José F. Cariñena and Arturo Ramos \\ Departamento de Física Teórica. Facultad de Ciencias. \\ Universidad de Zaragoza, 50009, Zaragoza, Spain.
}

\begin{abstract}
The basic concepts of factorizable problems in one-dimensional Quantum Mechanics, as well as the theory of Shape Invariant potentials are reviewed. The relation of this last theory with a generalization of the classical Factorization Method presented by Infeld and Hull is analyzed in detail. By the use of some properties of the Riccati equation the solutions of Infeld and Hull are greatly generalized in a simple way. PACS numbers: 11.30.Pb, 03.65.Fd.
\end{abstract}

\section{Introduction}

The Factorization Method introduced by Schrödinger [18, 19, 20] and later developed by Infeld and Hull [14] has been shown to be very efficient in the search of exactly solvable potentials and his interest has been increasing since the introduction by Witten of Supersymmetric Quantum Mechanics (SUSY) [26]. The bridge between the theory of solvable potentials in one dimension and SUSY was established by Gendenshteïn [8] who introduced the concept of a discrete reparametrization invariance, usually called "shape invariance". When studying all these related subjects one is really wondered by the almost complete ubiquity of some specific Riccati equations appearing in the theory. 
The Riccati equation, which is the simplest first order nonlinear differential equation, has a close relation with the group $S L(2, \mathbb{R})$ in the sense established in the celebrated, and unfortunately not so well known as it worths, Lie Scheffers theorem [15]. This theorem characterizes those first order differential equation systems admitting a nonlinear superposition principle. It is also known that the problem of finding the general solution for these systems is simplified from the knowledge of one or more particular solutions. All these aspects have been studied from a group theoretical perspective [1, 5] with special emphasis in the Riccati equation [3], which is nothing but the simplest prototype of equation having a nonlinear superposition principle (apart from the inhomogeneous linear equations, whose superposition principle reduces to a linear one).

We feel that an appropriate use of the mathematical properties of the Riccati equation may be very useful in order to obtain a deep insight in the theory of factorizable problems in Quantum Mechanics, as well as in its particular class given by Shape Invariant partner Hamiltonians.

To begin with, the mentioned properties can be used to obtain a simpler but more complete presentation, as well as a better understanding of the classical results given in [14. Indeed, we will prove that such results can be generalized by simply considering the general solution of certain Riccati equation instead of particular ones. In the end, all of the obtained solutions will give rise to specific, but rather general classes of Shape Invariant potentials in the sense of $[8]$.

Moreover, the techniques to be developed here can be very useful for attacking other still unsolved problems. For instance, one could consider the study of Shape Invariant potentials depending on several parameters transformed by translations as proposed in [6], which is the main subject of [4].

The paper is organized as follows: In Section 2 we review the theory of related operators and establish the concepts of partner potentials and Shape Invariant ones depending on an arbitrary set of parameters. In Section 3 we establish explicitly the equivalence between a slight generalization of the classical Factorization Method [14] and the theory of Shape Invariance. Section 4 is devoted to the study of an interesting differential equation system of key importance in the development of the subject. The first of its equations is a constant coefficients Riccati one for which we will find the solutions in full generality. We will use all these results in Section 5, where we obtain some rather general classes of factorizable problems which contain as par- 
ticular cases the classical results of [14]. In addition, these will give rise to several important families of Shape Invariant potentials which depend on one parameter transformed by translation.

\section{Hamiltonians related by first-order differ- ential operators. The concept of Shape In- variance.}

The problem of finding related operators having very similar spectra is now a well established subject (see e.g. [2] and references therein). Two linear differential operators $\tilde{H}$ and $H$ are said to be $A$-related if there exists an operator $A$ such that $A H=\tilde{H} A$, where $A$ need not to be invertible.

Furthermore, if we assume that the operator $A$ relating $H$ and $\tilde{H}$ is a first order differential operator, say, of the form

$$
A=\frac{d}{d x}+W(x)
$$

then, the relation $A H=\tilde{H} A$, with

$$
H=-\frac{d^{2}}{d x^{2}}+V(x), \quad \tilde{H}=-\frac{d^{2}}{d x^{2}}+\tilde{V}(x),
$$

leads to

$$
W(V-\tilde{V})=-W^{\prime \prime}-V^{\prime}, \quad V-\tilde{V}=-2 W^{\prime}
$$

while the relation $H A^{\dagger}=A^{\dagger} \tilde{H}$ leads to

$$
W(V-\tilde{V})=W^{\prime \prime}-\tilde{V}^{\prime}, \quad V-\tilde{V}=-2 W^{\prime} .
$$

One can easily integrate both pair of equations; from the first pair we obtain the equation $-2 W W^{\prime}=-W^{\prime \prime}-V^{\prime}$ and therefore

$$
V=W^{2}-W^{\prime}+c,
$$

with $c$ being an integrating constant. Following the same pattern with the second pair we have

$$
\tilde{V}=W^{2}+W^{\prime}+d,
$$


$d$ being also a constant. But taking into account $V-\tilde{V}=-2 W^{\prime}$ we have $c=d$. We have then the important property that two Hamiltonians $H$ and $\tilde{H}$ of the form given by (2) can be related by a first order differential operator $A$ given by (1) if and only if there exists a real constant $d$ such that $W$ satisfies the pair of Riccati equations

$$
\begin{aligned}
& V-d=W^{2}-W^{\prime} \\
& \tilde{V}-d=W^{2}+W^{\prime} .
\end{aligned}
$$

Moreover, this means that both Hamiltonians can be factorized as

$$
H=A^{\dagger} A+d, \quad \tilde{H}=A A^{\dagger}+d .
$$

Adding and subtracting equations (5) and (6) we obtain the equivalent pair which relates $V$ and $\tilde{V}$

$$
\begin{aligned}
\tilde{V}-d & =-(V-d)+2 W^{2} \\
\tilde{V} & =V+2 W^{\prime} .
\end{aligned}
$$

The potentials $\tilde{V}$ and $V$ are usually said to be partners. An important concept is the so called Shape Invariance introduced by Gendenshteïn [8]. He supposed that $V$ did depend on a certain set of parameters and considered the equations (5) and (6) as a definition of $V$ and $\tilde{V}$ in terms of a superpotential $W$. After, he asked himself what condition was necessary in order to get a partner $\tilde{V}$ of the same form as $V$ but for a different choice of the values of the parameters involved in $V$. This relation between $V$ and $\tilde{V}$ is now commonly known as Shape Invariance of the potentials [8].

More explicitly, we will suppose that our potentials are $V=V(x, a)$ and $\tilde{V}=\tilde{V}(x, a)$, where $a$ denotes a set of parameters. Gendenshtein [8] showed that if we assume the further relation between $V(x, a)$ and $\tilde{V}(x, a)$ given by

$$
\tilde{V}(x, a)=V(x, f(a))+R(f(a)),
$$

where $f$ is an (invertible and differentiable) transformation over the set of parameters $a$, then the complete spectrum of the Hamiltonians $H$ and $\tilde{H}$ can be found easily. Just writing the $a$-dependence the equations (5), (6) become

$$
\begin{aligned}
V(x, a)-d & =W^{2}-W^{\prime} \\
\tilde{V}(x, a)-d & =W^{2}+W^{\prime}
\end{aligned}
$$


The simplest way of satisfying these equations is assuming that $V(x, a)$ and $\tilde{V}(x, a)$ are obtained from a superpotential function $W(x, a)$ by means of

$$
\begin{aligned}
V(x, a)-d & =W^{2}(x, a)-W^{\prime}(x, a), \\
\tilde{V}(x, a)-d & =W^{2}(x, a)+W^{\prime}(x, a) .
\end{aligned}
$$

The Shape Invariance property in the sense of [8] requires the further condition (10) to be satisfied.

Let us remark that the parameter $a$ as well as the transformation law $f(a)$ are completely arbitrary up to now, apart from natural requirements as differentiability and invertibility. It is clear that the election of $a$ and $f(a)$ is what defines the different classes of Shape Invariant potentials. In principle, there is no reason why the intersection of these classes should be empty. We will consider a simple but important type in Section 5 .

\section{Equivalence between Shape Invariant po- tentials and the Factorization Method}

We consider in this section a slight generalization of the Factorization Method as appeared in the celebrated paper [14]. We will prove its equivalence with the theory of Shape Invariant partner potentials in the sense of [8].

Then, we will deal with the problem of factorizing the linear second-order ordinary differential equation

$$
\frac{d^{2} y}{d x^{2}}+r(x, a) y+\lambda y=0
$$

where the symbol $a$ denotes a set of $n$ independent real parameters, that is, $a=\left(a_{1}, \ldots, a_{n}\right)$. Let us consider a transformation on such parameter space $f(a)=\left(f_{1}(a), \ldots, f_{n}(a)\right)$. We will denote by $f^{k}$, where $k$ is a positive integer, the composition of $f$ with itself $k$ times. For a negative integer $k$ we will consider the composition of $f^{-1}$ with itself $k$ times and $f^{0}$ will be the identity. The admissible values of the parameters will be $f^{l}(a)$, where $l$ is an integer restricted to some subset to be precised later. The number $\lambda$ is in principle the eigenvalue to be determined. 
In a similar way as in [14], we will say that (15) can be factorized if it can be replaced by each of the two following equations:

$$
\begin{aligned}
H_{+}^{f^{-1}(a)} H_{-}^{f^{-1}(a)} y(\lambda, a) & =\left[\lambda-L\left(f^{-1}(a)\right)\right] y(\lambda, a), \\
H_{-}^{a} H_{+}^{a} y(\lambda, a) & =[\lambda-L(a)] y(\lambda, a),
\end{aligned}
$$

where

$$
H_{+}^{a}=\frac{d}{d x}+k(x, a), \quad H_{-}^{a}=-\frac{d}{d x}+k(x, a) .
$$

Here, $k(x, a)$ is a function to be determined which depends on the set of parameters $a$, and $L(a)$ is a real number for each value of the $n$-tuple $a$. The fundamental idea of this generalization is expressed in the following

Theorem 1 Let us suppose that our differential equation (15) can be factorized in the previously defined sense. If $y(\lambda, a)$ is one of its solutions then

$$
\begin{aligned}
y\left(\lambda, f^{-1}(a)\right) & =H_{-}^{f^{-1}(a)} y(\lambda, a) \\
y(\lambda, f(a)) & =H_{+}^{a} y(\lambda, a)
\end{aligned}
$$

are also solutions corresponding to the same $\lambda$ but to different values of the parameter $n$-tuple $a$, as it is suggested by the notations.

Proof.- Multiplying (16) by $H_{-}^{f^{-1}(a)}$ and (17) by $H_{+}^{a}$ we have

$$
\begin{gathered}
H_{-}^{f^{-1}(a)} H_{+}^{f^{-1}(a)} H_{-}^{f^{-1}(a)} y(\lambda, a)=\left[\lambda-L\left(f^{-1}(a)\right)\right] H_{-}^{f^{-1}(a)} y(\lambda, a), \\
H_{+}^{a} H_{-}^{a} H_{+}^{a} y(\lambda, a)=[\lambda-L(a)] H_{+}^{a} y(\lambda, a) .
\end{gathered}
$$

Comparison of these equations with (16) and (17) shows that $y\left(\lambda, f^{-1}(a)\right)$ as defined by (19) is a solution of (15) with $a$ replaced by $f^{-1}(a)$. Similarly $y(\lambda, f(a))$ given by (20) is a solution with $a$ replaced by $f(a)$.

It is to be remarked that (19) or (20) may give rise to the zero function; actually, we will see that this is necessary at some stage in order to obtain a sequence of square-integrable wave functions.

Indeed we are only interested here in square integrable solutions $y(\lambda, a)$. As we are dealing with one-dimensional problems, these solutions can be taken as real functions. Under this domain the following Theorem holds: 
Theorem 2 The linear operators $H_{+}^{a}$ and $H_{-}^{a}$ are formally mutually adjoint. That is, if $\phi \psi$ vanishes at the ends of the interval $I$,

$$
\int_{I} \phi\left(H_{-}^{a} \psi\right) d x=\int_{I} \psi\left(H_{+}^{a} \phi\right) d x
$$

Proof.- It is proved directly:

$$
\begin{aligned}
& \int_{I} \phi\left(H_{-}^{a} \psi\right) d x=-\int_{I} \phi \frac{d \psi}{d x} d x+\int_{I} \phi k(x, a) \psi d x \\
&=\int_{I} \psi \frac{d \phi}{d x} d x+\int_{I} \phi k(x, a) \psi d x=\int_{I} \psi\left(H_{+}^{a} \phi\right) d x,
\end{aligned}
$$

where we have integrated the first term by parts and used that $\psi \phi_{\mid \partial I}=0$.

Moreover, it is important to know when (19) and (20) produce new square-integrable functions.

Theorem 3 Let $y(\lambda, a)$ be a non-vanishing, square-integrable solution of (10) and (1X). The solution $y\left(\lambda, f^{-1}(a)\right)$ defined by (19) is square-integrable if and only if $\lambda \geq L\left(f^{-1}(a)\right)$. Similarly, the solution $y(\lambda, f(a))$ defined by (20) is square-integrable if and only if $\lambda \geq L(a)$.

Proof.- It is sufficient to compute

$$
\begin{aligned}
& \int_{I} y\left(\lambda, f^{-1}(a)\right)^{2} d x=\int_{I} H_{-}^{f^{-1}(a)} y(\lambda, a) H_{-}^{f^{-1}(a)} y(\lambda, a) d x \\
& =\int_{I} y(\lambda, a)\left(H_{+}^{f^{-1}(a)} H_{-}^{f^{-1}(a)} y(\lambda, a)\right) d x=\left(\lambda-L\left(f^{-1}(a)\right)\right) \int_{I} y(\lambda, a)^{2} d x
\end{aligned}
$$

where it has been used Theorem 2 and (16). In a similar way,

$$
\begin{aligned}
& \int_{I} y(\lambda, f(a))^{2} d x=\int_{I} H_{+}^{a} y(\lambda, a) H_{+}^{a} y(\lambda, a) d x \\
& =\int_{I} y(\lambda, a)\left(H_{-}^{a} H_{+}^{a} y(\lambda, a)\right) d x=(\lambda-L(a)) \int_{I} y(\lambda, a)^{2} d x,
\end{aligned}
$$

where use has been made of Theorem 2 and (17).

We will consider now the sequence $L\left(f^{k}(a)\right)$ and analyze only the cases where it is either an increasing or a decreasing sequence. A more complicated behavior of $L\left(f^{k}(a)\right)$ with $k$ (e.g. oscillatory) will not be treated here. 
Theorem 4 Suppose that $L\left(f^{k}(a)\right)$ is a decreasing sequence with no accumulation points. Then the necessary and sufficient condition for having squareintegrable solutions of the equations (10) and (17) is that there exists a point of the parameter space, $b=\left(b_{1}, \ldots, b_{n}\right)$, such that

$$
\lambda=L(b), \quad H_{-}^{b} y(\lambda, f(b))=0,
$$

provided the function $y(L(b), f(b))$ so obtained is square-integrable.

Proof.- Let $y(\lambda, a)$ be a non-vanishing, square-integrable solution of (16) and (17). In order to avoid a contradiction it is necessary, by Theorem [3, that $\lambda \geq L\left(f^{-1}(a)\right)$. If the equality does not hold, one can iterate the process to obtain

$$
\int_{I} y\left(\lambda, f^{-2}(a)\right)^{2} d x=\left(\lambda-L\left(f^{-2}(a)\right)\right)\left(\lambda-L\left(f^{-1}(a)\right)\right) \int_{I} y(\lambda, a)^{2} d x .
$$

Since $L\left(f^{k}(a)\right)$ is decreasing with $k$, we have that the difference $\lambda-L\left(f^{-2}(a)\right)$ is positive or vanishing and smaller than $\lambda-L\left(f^{-1}(a)\right)$. If it still does not vanish, the process can be continued until we arrive at a value $k_{0}$ such that $\lambda=$ $L\left(f^{-k_{0}}(a)\right)$. It is then necessary that $y\left(\lambda, f^{-k_{0}}(a)\right)=H_{-}^{f^{-k_{0}}(a)} y\left(\lambda, f^{-k_{0}+1}(a)\right)=$ 0 . It suffices to set $b=f^{-k_{0}}(a)$ to obtain the result.

Theorem 5 If $L\left(f^{k}(a)\right)$ is an increasing sequence with no accumulation points, then the necessary and sufficient condition for having square-integrable solutions of the equations (16) and (17) is that there exists a specific point of the parameter space, $b=\left(b_{1}, \ldots, b_{n}\right)$, such that

$$
\lambda=L(b), \quad H_{+}^{b} y(\lambda, b)=0,
$$

provided the function $y(L(b), b)$ so obtained is square-integrable.

Proof.- Let $y(\lambda, a)$ be a non-vanishing, square-integrable solution of (16) and (17). In order to avoid a contradiction it is necessary by Theorem 3 that $\lambda \geq L(a)$. If the equality does not hold, one can iterate the process to obtain

$$
\int_{I} y\left(\lambda, f^{2}(a)\right)^{2} d x=(\lambda-L(f(a)))(\lambda-L(a)) \int_{I} y(\lambda, a)^{2} d x .
$$


Since $L\left(f^{k}(a)\right)$ is an increasing sequence, $\lambda-L(f(a))$ is positive or vanishing and smaller than $\lambda-L(a)$. If it still does not vanish, the process can be continued until we arrive at $k_{0}$ such that $\lambda=L\left(f^{k_{0}-1}(a)\right)$. Then, it is necessary $y\left(\lambda, f^{k_{0}}(a)\right)=H_{+}^{f^{k_{0}-1}(a)} y\left(\lambda, f^{k_{0}-1}(a)\right)=0$. It suffices to set $b=f^{k_{0}-1}(a)$.

When $L\left(f^{k}(a)\right)$ is a decreasing (resp. increasing) sequence, the functions $y$ defined by $H_{-}^{b} y(L(b), f(b))=0\left(\right.$ resp. $\left.H_{+}^{b} y(L(b), b)=0\right)$, provided they are square-integrable, will be those from where all the others will be constructed.

We consider now what relation among $r(x, a), k(x, a)$ and $L(a)$ there exists. Carrying out explicitly the calculations involved in (16) and (17), using (15), we find the equations

$$
\begin{gathered}
k^{2}\left(x, f^{-1}(a)\right)+\frac{d k\left(x, f^{-1}(a)\right)}{d x}=-r(x, a)-L\left(f^{-1}(a)\right), \\
k^{2}(x, a)-\frac{d k(x, a)}{d x}=-r(x, a)-L(a) .
\end{gathered}
$$

We can eliminate $r(x, a)$ between these equations, obtaining

$$
k^{2}\left(x, f^{-1}(a)\right)-k^{2}(x, a)+\frac{d k\left(x, f^{-1}(a)\right)}{d x}+\frac{d k(x, a)}{d x}=L(a)-L\left(f^{-1}(a)\right) .
$$

Moreover, since (24) and (25) hold for each $f^{k}(a), k$ in the range of integers corresponding to square-integrable solutions, we can rewrite them as

$$
\begin{aligned}
& k^{2}(x, a)+\frac{d k(x, a)}{d x}=-r(x, f(a))-L(a), \\
& k^{2}(x, a)-\frac{d k(x, a)}{d x}=-r(x, a)-L(a),
\end{aligned}
$$

and from them we can obtain the equivalent pair

$$
\begin{aligned}
& r(x, a)+r(x, f(a))+2 k^{2}(x, a)+2 L(a)=0, \\
& r(x, a)-r(x, f(a))-2 \frac{d k(x, a)}{d x}=0 .
\end{aligned}
$$

Both of the equations (24) and (25) are necessary conditions to be satisfied by $k(x, a)$ and $L(a)$, for a given $r(x, a)$. They are also sufficient since any $k(x, a)$ and $L(a)$ satisfying these equations lead unambiguously to a function $r(x, a)$ and so to a problem whose factorization is known. It should be noted, 
however, that there exists the possibility that equations (24) and (25) did not have in general a unique solution for $k(x, a)$ and $L(a)$ for a given $r(x, a)$.

The equation (26) is what one uses in practice in order to obtain results of the Factorization Method. We try to solve (26) instead of (24) and (25) since is easier to find problems which are factorizable by construction than seeing whether certain problem defined by some $r(x, a)$ is factorizable or not.

Conversely, a solution $k(x, a)$ of (26) gives rise to unique expressions for the differences $-r(x, f(a))-L(a)$ and $-r(x, a)-L(a)$ by means of equations (27) and (28), but it does not determine the quantities $r(x, a)$ and $L(a)$ in a unique way. In fact, the method does not determine the function $L(a)$ unambiguously but only the difference $L(f(a))-L(a)$. And this does not define $L(a)$ in a unique way at all. To begin with, $L(a)$ is always defined up to a constant. And more ambiguity could arise in some cases, as it happens in the case studied in [4]. But for the purposes of the application of this method to Quantum Mechanics the interesting quantity is $L(f(a))-L(a)$, as we will see below. The same way is undetermined $r(x, a)$, with an ambiguity which cancels out exactly with that of $L(a)$ since the differences $-r(x, f(a))-L(a)$ and $-r(x, a)-L(a)$ are completely determined from a given solution $k(x, a)$ of (26).

Going back to the problem of finding Shape Invariant potentials in the sense of [8] which depend on the same set of parameters $a$, we remember that the equations to be satisfied are (13) and (14) or the equivalent equations

$$
\begin{aligned}
\tilde{V}(x, a)-d & =-(V(x, a)-d)+2 W^{2}(x, a), \\
\tilde{V}(x, a) & =V(x, a)+2 W^{\prime}(x, a)
\end{aligned}
$$

as well as the Shape Invariance condition (10).

Remember that the potentials $V(x, a)$ and $\tilde{V}(x, a)$ define a pair of Hamiltonians

$$
H(a)=-\frac{d^{2}}{d x^{2}}+V(x, a), \quad \tilde{H}(a)=-\frac{d^{2}}{d x^{2}}+\tilde{V}(x, a),
$$

which can be factorized as

$$
H(a)=A(a)^{\dagger} A(a)+d, \quad \tilde{H}(a)=A(a) A(a)^{\dagger}+d,
$$

where $d$ is a real number and

$$
A(a)=\frac{d}{d x}+W(x, a), \quad A^{\dagger}(a)=-\frac{d}{d x}+W(x, a) .
$$


The Shape Invariance condition reads in terms of these Hamiltonians

$$
\tilde{H}(a)=H(f(a))+R(f(a)) .
$$

We establish next the identifications between the symbols used in the generalized Factorization Method treated in this section and those used in the theory of Shape Invariance. We will see that the equations to be satisfied are exactly the same, and that both problems essentially coincide when we consider square-integrable solutions. For that purpose is sufficient to identify

$$
\begin{aligned}
\tilde{V}(x, a)-d & =-r(x, f(a))-L(a), \\
V(x, a)-d & =-r(x, a)-L(a) \\
W(x, a) & =k(x, a) \\
R(f(a)) & =L(f(a))-L(a),
\end{aligned}
$$

and as an immediate consequence,

$$
A(a)=H_{+}^{a}, \quad A^{\dagger}(a)=H_{-}^{a},
$$

for all allowed values of $a$. In fact, with these identifications it is immediate to see that equations (27) and (28) are equivalent to (14) and (13), respectively. Moreover

$$
\begin{aligned}
\tilde{V}(x, a)-V(x, f(a)) & =-r(x, f(a))-L(a)+r(x, f(a))+L(f(a)) \\
& =L(f(a))-L(a)=R(f(a))
\end{aligned}
$$

which is nothing but equation (10); equations (29), (30) become

$$
\begin{aligned}
& -(V(x, a)-d)-L(a)-(\tilde{V}(x, a)-d)-L(a)+2 W^{2}(x, a)+2 L(a) \\
& \quad=-(V(x, a)-d)-(\tilde{V}(x, a)-d)+2 W^{2}(x, a)=0,
\end{aligned}
$$

and

$$
\begin{aligned}
& -(V(x, a)-d)-L(a)+(\tilde{V}(x, a)-d)+L(a)-2 W^{\prime}(x, a) \\
& =-V(x, a)+\tilde{V}(x, a)-2 W^{\prime}(x, a)=0,
\end{aligned}
$$

i.e., equations (31) and (32), respectively.

But the identification does not stop here. Let us assume that Theorem 4 is applicable. We shall see what it means in terms of the Hamiltonians (34). To begin with, we have a certain point of the parameter space 
$b=\left(b_{1}, \ldots, b_{n}\right)$ such that $\lambda=L(b)$ and $A^{\dagger}(b) y(L(b), f(b))=0$, where the function $y(L(b), f(b))$ so defined is square-integrable. We will omit its first argument for brevity, writing $y(f(b))$. It is given by the expression

$$
y(f(b))=N \exp \left(\int^{x} W(\xi, b) d \xi\right),
$$

where $N$ is a normalization constant. Note that this wave function has no nodes. Since $L\left(f^{k}(a)\right)$ is a decreasing sequence, we have that the function $R\left(f^{k}(b)\right)=L\left(f^{k}(b)\right)-L\left(f^{k-1}(b)\right)<0$ for all of the acceptable values of $k$.

Then, it is easy to check that $y(f(b))$ is the ground state of the Hamiltonian $\tilde{H}(b)$, with energy $d$. In fact,

$$
\tilde{H}(b) y(f(b))=\left(A(b) A(b)^{\dagger}+d\right) y(f(b))=d y(f(b)) .
$$

From equation (36) we have $H(b)=\tilde{H}\left(f^{-1}(b)\right)-R(b)$. The function $y(b)$ is the ground state of $H(b)$ with energy $d-R(b)$ :

$$
H(b) y(b)=\tilde{H}\left(f^{-1}(b)\right) y(b)-R(b) y(b)=(d-R(b)) y(b) .
$$

Now, the first excited state of $\tilde{H}(b)$ is $A(b) y(b)$ :

$$
\tilde{H}(b) A(b) y(b)=A(b) H(b) y(b)=(d-R(b)) A(b) y(b),
$$

where it has been used the property $\tilde{H}(b) A(b)=A(b) H(b)$. In a similar way it can be proved that $A\left(f^{-1}(b)\right) y\left(f^{-1}(b)\right)$ is the first excited state of $H(b)$, with energy $d-R(b)-R\left(f^{-1}(b)\right)$. One can iterate the procedure in order to solve completely the eigenvalue problem of the Hamiltonians $H(b)$ and $\tilde{H}(b)$. The results are summarized in Table 1 . Note that $d$ has the meaning of the reference energy chosen for the Hamiltonians. It is usually taken as zero.

A similar pattern can be followed when it is applicable the Theorem 5 , that is, when $L\left(f^{k}(a)\right)$ is an increasing sequence. The results are essentially the same as when the sequence is decreasing but where now the Hamiltonian with a lower ground state energy is $H(b)$. The basic square-integrable eigenfunction $y(b)$ is defined now by $A(b) y(b)=0$, that is,

$$
y(b)=M \exp \left(-\int^{x} W(\xi, b) d \xi\right),
$$

where $M$ is the normalization constant. Moreover, now $R\left(f^{k}(b)\right)>0$ for all of the acceptable values of $k$. The results are summarized in Table 2. Again, $d$ sets the energy reference level of the Hamiltonians. 


\begin{tabular}{|c|c|c|}
\hline Eigenfunctions and energies & $\tilde{H}(b)$ & $H(b)$ \\
\hline \multirow{2}{*}{ Ground state } & $y(f(b))$ & $y(b)$ \\
& $d$ & $d-R(b)$ \\
\hline$k^{\text {th }}$ excited state & $A(b) \cdots A\left(f^{-k+1}(b)\right) y\left(f^{-k+1}(b)\right)$ & $A\left(f^{-1}(b)\right) \cdots A\left(f^{-k}(b)\right) y\left(f^{-k}(b)\right)$ \\
& $d-\sum_{r=0}^{k-1} R\left(f^{-r}(b)\right)$ & $d-\sum_{r=0}^{k} R\left(f^{-r}(b)\right)$ \\
\hline
\end{tabular}

Table 1: Eigenfunctions and eigenvalues of $\tilde{H}(b)$ and $H(b)$ when Theorem 4 is applicable. The function $y(f(b))$ is defined by the relation $A^{\dagger}(b) y(f(b))=0$.

\begin{tabular}{|c|c|c|}
\hline Eigenfunctions and energies & $H(b)$ & \multicolumn{1}{|c|}{$\tilde{H}(b)$} \\
\hline \multirow{2}{*}{ Ground state } & $y(b)$ & $y(f(b))$ \\
& $d$ & $d+R(f(b))$ \\
\hline$k^{\text {th }}$ excited state & $A^{\dagger}(b) \cdots A^{\dagger}\left(f^{k-1}(b)\right) y\left(f^{k}(b)\right)$ & $A^{\dagger}(f(b)) \cdots A^{\dagger}\left(f^{k}(b)\right) y\left(f^{k+1}(b)\right)$ \\
& $d+\sum_{r=1}^{k} R\left(f^{r}(b)\right)$ & $d+\sum_{r=1}^{k+1} R\left(f^{r}(b)\right)$ \\
\hline
\end{tabular}

Table 2: Eigenfunctions and eigenvalues of $H(b)$ and $\tilde{H}(b)$ when is applicable Theorem 5. The function $y(b)$ is defined by the relation $A(b) y(b)=0$. 
In both cases the spectra of both Hamiltonians are exactly the same (with corresponding eigenfunctions shifted in one step) except for the ground state of one of them, which has the lowest possible energy. Only one of the eigenfunctions, either (42) or (43) may be square-integrable. It might happen, however, that neither of these functions were so. In such a situation none of the schemes we have developed would be of use. The conditions on the function $W(x, b)$ such that one of the possible ground states exist are explained e.g. in [9]. Essentially it depends on the asymptotic behavior of $\int^{x} W(\xi, b) d \xi$ as $x \rightarrow \pm \infty$.

In view of all of these identifications the following result is stated

Theorem 6 The problem of finding the square integrable solutions of the factorization of (15), given by equations (10) and (17), is the same as to solve the discrete eigenvalue problem of the Shape Invariant Hamiltonians (34) in the sense of [8] which depend on the same set of parameters.

We encourage the reader to compare the results obtained in this section with the ones in [14, pp. 24-27], which have inspired this generalization.

Let us consider now the simplest but particularly important case of having only one parameter whose transformation law is a translation, that is,

$$
f(a)=a-\epsilon, \quad \text { or } \quad f(a)=a+\epsilon,
$$

where $\epsilon \neq 0$. In both cases we can normalize the parameter in units of $\epsilon$, introducing the new parameter

$$
m=\frac{a}{\epsilon}, \quad \text { or } \quad m=-\frac{a}{\epsilon},
$$

respectively. In each of these two possibilities the transformation law reads, with a slight abuse of the notation $f$,

$$
f(m)=m-1 .
$$

Then, the equations to be solved for finding Shape Invariant potentials, in the sense of [8], depending on one parameter transformed by a translation are

$$
\begin{aligned}
V(x, m)-d & =W^{2}(x, m)-W^{\prime}(x, m), \\
\tilde{V}(x, m)-d & =W^{2}(x, m)+W^{\prime}(x, m),
\end{aligned}
$$


or the equivalent equations

$$
\begin{aligned}
\tilde{V}(x, m)-d & =-(V(x, m)-d)+2 W^{2}(x, m), \\
\tilde{V}(x, m) & =V(x, m)+2 W^{\prime}(x, m),
\end{aligned}
$$

as well as the Shape Invariance condition

$$
\tilde{V}(x, m)=V(x, m-1)+R(m-1) .
$$

As a particular case of Theorem 6 we have the following

Corollary 1 The problem of finding all factorizable problems following the Factorization Method stated in [14] is equivalent to find Shape Invariant potentials in the sense of [8] which depend on one parameter transformed by translation.

The relations among the relevant quantities in both approaches are given next for completeness,

$$
\begin{aligned}
\tilde{V}(x, m)-d & =-r(x, m-1)-L(m), \\
V(x, m)-d & =-r(x, m)-L(m), \\
W(x, m) & =k(x, m) . \\
R(m-1) & =L(m-1)-L(m) .
\end{aligned}
$$

We would like to remark that the equivalence between the Factorization Method and Shape Invariance has been first pointed out, to our knowledge, by several authors almost ten years ago (see e.g. [22, 23] and [16]). It seems to us that most of the authors in these subjects have the feeling (or even a more precise knowledge) that such identification exists. But we have not seen so far a complete and clear identification in the general case where arbitrary set of parameters $a$ and transformation laws $f(a)$ are involved. Our aim is just to take a step ahead in the task of clarifying how these methods are interrelated since they can be used in more general situations. An important example of this is obtained when an arbitrary but finite number of parameters subject to translation is involved [4]. 


\section{General solution of equations $y^{2}+y^{\prime}=a$, $z y+z^{\prime}=b$}

In this section we will study the general solution of an ordinary differential equation system which will appear as the key point in the solution of the problems posed in [14, which we will revisit in the next section.

Let us consider the differential equation system in the variables $y$ and $z$

$$
\begin{aligned}
& y^{2}+y^{\prime}=a, \\
& y z+z^{\prime}=b,
\end{aligned}
$$

where $a$ and $b$ are real constants and the prime denotes derivative respect to $x$. The equation (56) is a Riccati equation with constant coefficients, meanwhile (57) is an inhomogeneous linear first order differential equation

for $z$, provided the function $y$ is known. Recall that the general solution of the inhomogeneous linear first order differential equation for $v(x)$

$$
\frac{d v}{d x}=a(x) v(x)+b(x)
$$

can be obtained by means of the formula

$$
v(x)=\frac{\int^{x} b(\xi) \exp \left\{-\int^{\xi} a(\eta) d \eta\right\} d \xi+E}{\exp \left\{-\int^{x} a(\xi) d \xi\right\}},
$$

where $E$ is an integration constant. Then, the general solution of (57) is easily obtained once we know the solutions of (56), i.e.

$$
z(x)=\frac{b \int^{x} \exp \left\{\int^{\xi} y(\eta) d \eta\right\} d \xi+D}{\exp \left\{\int^{x} y(\xi) d \xi\right\}},
$$

where we name the integration constant as $D$. So, let us first pay attention to the task of solving (56) in its full generality.

The general Riccati equation

$$
\frac{d y}{d x}=a_{2}(x) y^{2}+a_{1}(x) y+a_{0}(x)
$$

where $a_{2}(x), a_{1}(x)$ and $a_{0}(x)$ are differentiable functions of the independent variable $x$, has very interesting properties. We will recall here some of them 
which will be of use in our problem. It is a non-linear first order differential equation, and in the most general case there is no way of writing the general solution by using some quadratures. However, one can integrate it completely if some extra information is known. For example, if one particular solution $y_{1}(x)$ of (61) is known, the problem can be reduced to an inhomogeneous first order linear equation and the general solution can be found by two quadratures. In fact, the change of variable (see e.g. [7, 17])

$$
u=\frac{1}{y_{1}-y}, \quad \text { with inverse } \quad y=y_{1}-\frac{1}{u}
$$

transforms (61) into the inhomogeneous first order linear equation

$$
\frac{d u}{d x}=-\left(2 a_{2} y_{1}+a_{1}\right) u+a_{2},
$$

which can be integrated by two quadratures, for example using (59). An alternative change of variable was also found in [3]:

$$
u=\frac{y y_{1}}{y_{1}-y}, \quad \text { with inverse } \quad y=\frac{u y_{1}}{u+y_{1}} .
$$

This change transforms (61) into the inhomogeneous first order linear equation

$$
\frac{d u}{d x}=\left(\frac{2 a_{0}}{y_{1}}+a_{1}\right) u+a_{0}
$$

which is integrable by two quadratures, as well. We also remark that the general Riccati equation (61) admits the identically vanishing function as a solution if and only if $a_{0}(x)=0$ for all $x$.

Even more interesting is the following property: once three particular solutions of (61), $y_{1}(x), y_{2}(x), y_{3}(x)$, are known, the general solution $y$ can be written, without making use of any quadrature, by means of the formula

$$
\frac{\left(y-y_{1}\right)\left(y_{2}-y_{3}\right)}{\left(y-y_{2}\right)\left(y_{1}-y_{3}\right)}=k
$$

where $k$ is a constant determining each solution. Solving for $y$ we get

$$
y=\frac{y_{2}\left(y_{3}-y_{1}\right) k+y_{1}\left(y_{2}-y_{3}\right)}{\left(y_{3}-y_{1}\right) k+y_{2}-y_{3}} .
$$


As an example, it is easy to check that $\left.y\right|_{k=0}=y_{1},\left.y\right|_{k=1}=y_{3}$ and that the solution $y_{2}$ is obtained as the limit of $k$ going to $\infty$.

The theorem for uniqueness of solutions of differential equations shows that the difference between two solutions of the Riccati equation (61) has a constant sign and therefore the difference between two different solutions never vanishes, and the quotients in the previous equations are always well defined.

The equation (67) furnishes a non-linear superposition principle for the Riccati equation: there exists a superposition function $\Phi\left(u_{1}, u_{2}, u_{3}, k\right)$ such that for any three particular fundamental solutions, the function $\Phi\left(y_{1}, y_{2}, y_{3}, k\right)$ gives the general solution.

The first order differential equation systems having this important property are characterized by the so called Lie-Scheffers theorem 15], the simplest one being the Riccati equation (apart from the inhomogeneous first order linear equation, whose superposition principle reduces to a linear one). These problems have had a revival after several interesting papers by Winternitz and coworkers (see e.g. 24] and references therein), and have been studied in [1] from a group theoretical perspective. In [3] the integrability conditions of the Riccati equation, as well as its non-linear superposition principle are studied in a unified way by making use of an action on the set of Riccati equations. A generalization to other groups and systems admitting such a non-linear superposition principle is given in [5].

We are interested here in the simpler case of the Riccati equation with constant coefficients (56). The general equation of this type is

$$
\frac{d y}{d x}=a_{2} y^{2}+a_{1} y+a_{0}
$$

where $a_{2}, a_{1}$ and $a_{0}$ are now real constants, $a_{2} \neq 0$. For a review of some of its properties from a geometrical viewpoint see [3]. This equation, unlike the general Riccati equation (61), is always integrable by quadratures, and the form of the solutions depends strongly on the sign of the discriminant $\Delta=a_{1}^{2}-4 a_{0} a_{2}$. This can be seen by separating the differential equation (68) in the form

$$
\frac{d y}{a_{2} y^{2}+a_{1} y+a_{0}}=\frac{d y}{a_{2}\left(\left(y+\frac{a_{1}}{2 a_{2}}\right)^{2}-\frac{\Delta}{4 a_{2}^{2}}\right)}=d x .
$$

Integrating (68) in this way we obtain non-constant solutions. 
Looking for constant solutions of (68) amounts to solve an algebraic second order equation. So, if $\Delta>0$ there will be two different real constant solutions. If $\Delta=0$ there is only one constant real solution and if $\Delta<0$ we have no constant real solutions at all.

We shall illustrate these properties while finding the general solution of (56). For this equation the discriminant $\Delta$ is just $4 a$. Then, the form of the solutions depend strongly on the sign of $a$. If $a>0$ we can write $a=c^{2}$, where $c>0$ is a real number. The non-constant particular solution

$$
y_{1}(x)=c \tanh (c(x-A)),
$$

where $A$ is an arbitrary integration constant, is readily found by direct integration. In addition, there exists two different constant real solutions,

$$
y_{2}(x)=c, \quad y_{3}(x)=-c
$$

Then, we can find out the general solution from these particular solutions using the non-linear superposition formula (67), yielding

$$
y(x)=c \frac{B \sinh (c(x-A))-\cosh (c(x-A))}{B \cosh (c(x-A))-\sinh (c(x-A))},
$$

where $B=(2-k) / k, k$ being the arbitrary constant in (67). Substituting in (60) we obtain the general solution for $z(x)$,

$$
z(x)=\frac{\frac{b}{c}\{B \sinh (c(x-A))-\cosh (c(x-A))\}+D}{B \cosh (c(x-A))-\sinh (c(x-A))},
$$

where $D$ is a new integration constant.

Let us study now the case with $a=0$ in (56). By direct integration we find the particular solution

$$
y_{1}(x)=\frac{1}{x-A}
$$

where $A$ is an integration constant. It is clear that now (56) admits the identically vanishing solution, and the general solution have to reflect this fact. In order to find it is particularly simple the application of the change of variable (64) with $y_{1}$ given by (73). Indeed, such a change transforms (56) 
with $a=0$ into $d u / d x=0$, which has the general solution $u(x)=B, B$ constant. Then, the general solution for (56) with $a=0$ is

$$
y(x)=\frac{B}{1+B(x-A)},
$$

with $A$ and $B$ being arbitrary integration constants. If $B=0$ we recover the identically vanishing solution as expected. Had we followed the usual change of variable (62) we would have obtained exactly the same result, but the calculations would have been a bit longer. Substituting in (60) we obtain the general solution for $z(x)$ in this case,

$$
z(x)=\frac{b\left(\frac{B}{2}(x-A)^{2}+x-A\right)+D}{1+B(x-A)},
$$

where $D$ is a new integration constant.

The last case to be studied is $a<0$. We write then $a=-c^{2}$, where $c>0$ is a real number. It is easy to find the non-constant particular solution

$$
y_{1}(x)=-c \tan (c(x-A)),
$$

where $A$ is an arbitrary integration constant, by direct integration. In order to find out the general solution, we make the change of variable (62) or alternatively (64), with $y_{1}(x)$ given by $(\sqrt{76})$. In both cases the calculations are essentially the same and give the general solution of (56) for $a>0$

$$
y(x)=-c \frac{B \sin (c(x-A))+\cos (c(x-A))}{B \cos (c(x-A))-\sin (c(x-A))},
$$

where $B=c F, F$ an arbitrary constant. Substituting in (60) we obtain the corresponding general solution for $z(x)$,

$$
z(x)=\frac{\frac{b}{c}\{B \sin (c(x-A))+\cos (c(x-A))\}+D}{B \cos (c(x-A))-\sin (c(x-A))},
$$

where $D$ is a new integration constant.

Needless to say, in all of the three cases the solutions can be written in many ways, mostly in the cases where exponential, hyperbolic or trigonometric functions are involved. The choice of the form in which the arbitrary constants appear might also make the solutions to look a bit different, but 
these aspects are irrelevant from the mathematical point of view. We have tried to give the simplest form for the solutions and in such a way the symmetry between the solutions for the case $a>0$ and $a<0$ were clearly recognized. Indeed, the general solution of (56) for $a>0$ can be transformed into that of the case $a<0$ by means of the formal changes $c \rightarrow i c, B \rightarrow i B$ and the identities $\sinh (i x)=i \sin (x), \cosh (i x)=\cos (x)$. The change for $B$ is motivated by its definition in the the general solution of (56) for $a<0$. The results are summarized in Table 3 .

We must pay attention to the following point. If we consider, for instance, the general solution of (56) for $a>0$, i.e. equation (71), one could be tempted to write it in the form of a logarithmic derivative,

$$
y(x)=\frac{d}{d x} \log |B \cosh (c(x-A))-\sinh (c(x-A))|,
$$

which is equivalent except for $B \rightarrow \infty$. In fact, if we want to calculate

$$
\lim _{B \rightarrow \infty} \frac{d}{d x} \log |B \cosh (c(x-A))-\sinh (c(x-A))|
$$

we cannot interchange the limit with the derivative, otherwise we would get a wrong result. The reason, obviously, is that $B \cosh (c(x-A))-\sinh (c(x-A))$ is not regular as $B \rightarrow \infty$. But this limit for $B$ is particularly important since when taking it in (71), we recover the particular solution (69). A similar thing happens in the general solutions (74) and (77), where after taking the limit $B \rightarrow \infty$ we recover, respectively, the particular solutions (73) and (76) from which we have started. Both of (74) and (77) can be written in the form of a logarithmic derivative, but then the limit $B \rightarrow \infty$ could not be calculated properly.

The conclusion is the following. If one or more particular solutions of a Riccati equation are known, the general solution can be found, for example, by one of the methods described above. This general solution depends on one parameter characterizing the particular solutions, and in particular one should be able to recover the known solutions for some specific values. One of these values is usually infinite. If one writes the general solution as a logarithmic derivative, the limit when the parameter tends to infinite is to be treated with care. 


\begin{tabular}{|l|c|c|}
\hline \multicolumn{1}{|c|}{ Sign of $a$} & $y(x)$ & $z(x)$ \\
\hline$a=c^{2}>0$ & $c \frac{B \sinh (c(x-A))-\cosh (c(x-A))}{B \cosh (c(x-A))-\sinh (c(x-A))}$ & $\frac{\frac{b}{c}\{B \sinh (c(x-A))-\cosh (c(x-A))\}+D}{B \cosh (c(x-A))-\sinh (c(x-A))}$ \\
\hline$a=0$ & $\frac{B}{1+B(x-A)}$ & $\frac{b\left(\frac{B}{2}(x-A)^{2}+x-A\right)+D}{1+B(x-A)}$ \\
\hline$a=-c^{2}<0$ & $-c \frac{B \sin (c(x-A))+\cos (c(x-A))}{B \cos (c(x-A))-\sin (c(x-A))}$ & $\frac{\frac{b}{c}\{B \sin (c(x-A))+\cos (c(x-A))\}+D}{B \cos (c(x-A))-\sin (c(x-A))}$ \\
\hline
\end{tabular}

Table 3: General solutions of the equations (56) and (57). $A, B$ and $D$ are integration constants. The constant $B$ selects the particular solution of (56) in each case.

\section{The Infeld-Hull Factorization Method re- visited: Shape Invariant potentials depend- ing on one parameter transformed by trans- lation}

We will start this section reviewing the steps of the famous paper [14], where the Factorization Method was developed in a quite systematic way. It is worth mentioning, however, that this method take its roots on previous papers by Schrödinger [18, 19, 20] and others (see references in [14, p. 23]). We will apply the mathematical theory developed in the preceding sections for solving the problem in a simple way and with full generality, obtaining in the end Shape-Invariant potentials in the sense of 8 depending on one parameter transformed by translation.

The key point in the process of finding factorizable problems of type (15) is to find solutions $k(x, a)$ for the equation (26), as we have said in Section 3 . In our current problem it takes the form

$$
k^{2}(x, m+1)-k^{2}(x, m)+\frac{d k(x, m+1)}{d x}+\frac{d k(x, m)}{d x}=L(m)-L(m+1),
$$

which is a differential--difference equation. The idea of solving it in its full 
generality seems to be very difficult, at least at first sight. Instead of doing that, it seems to be more sensible to try particular forms of the dependence of $k(x, m)$ on $x$ and $m$. Then, we should find out whether the equation is satisfied in each particular case.

First, note (see [14]) that there exists a trivial solution of (79), namely

$$
k(x, m)=f(m), \quad L(m)=-f^{2}(m),
$$

where $f(m)$ is any function of $m$. This gives rise to the problem

$$
\frac{d^{2} y}{d x^{2}}+\lambda y=0
$$

which has been discussed completely by Schrödinger [19].

We next try a solution with an affine dependence on $m$ [14]

$$
k(x, m)=k_{0}(x)+m k_{1}(x),
$$

where $k_{0}$ and $k_{1}$ are functions of $x$ only. Substituting into $(79)$ we obtain the equation

$$
\begin{aligned}
L(m)-L(m+1)= & {\left[(m+1)^{2}\left(k_{1}^{2}+k_{1}^{\prime}\right)+2(m+1)\left(k_{0} k_{1}+k_{0}^{\prime}\right)\right] } \\
& -\left[m^{2}\left(k_{1}^{2}+k_{1}^{\prime}\right)+2 m\left(k_{0} k_{1}+k_{0}^{\prime}\right)\right] .
\end{aligned}
$$

Now we would like to reinterpret the reasoning followed in [14, p.27]. Equation (81) reads in its more simplified way

$$
L(m)-L(m+1)=2 m\left(k_{1}^{2}+k_{1}^{\prime}\right)+k_{1}^{2}+k_{1}^{\prime}+2\left(k_{0} k_{1}+k_{0}^{\prime}\right) .
$$

Since $L(m)$ is a function of $m$ alone, the coefficients of the powers of $m$ on the right hand side must be constant. Eventually one finds the same coefficients to be constant as in the equation appearing after (3.1.4) of [14. Then, the equations to be satisfied are

$$
\begin{aligned}
k_{1}^{2}+k_{1}^{\prime} & =a, \\
k_{1} k_{0}+k_{0}^{\prime} & =b,
\end{aligned}
$$

where $a$ and $b$ are in principle real arbitrary constants. When these equations are satisfied (82) becomes

$$
L(m)-L(m+1)=2(m a+b)+a .
$$


We look for the most general polynomial solution of this equation. It should be of degree two in $m$ if $a \neq 0$ (degree one if $a=0$ ); otherwise we would find that the coefficients of powers greater or equal to three (resp. two) have to vanish. Then we put $L(m)=r m^{2}+s m+t$, where $r, s, t$ are constants to be determined. Substituting in the previous equation we find the relations

$$
r=-a, \quad s=-2 b,
$$

and as a result we have the most general polynomial solution for $L(m)$

$$
L(m)=-a m^{2}-2 b m+t,
$$

where $t$ is an arbitrary real constant. This expression is valid even in the case $a=0$, being then $L(m)=-2 b m+t$.

In [14, eqs. (3.1.5)] equations (83), (84) are written in the slightly more restricted way (we use Greek characters to avoid confusion)

$$
\begin{aligned}
k_{1}^{2}+k_{1}^{\prime} & =-\alpha^{2}, \\
k_{1} k_{0}+k_{0}^{\prime} & =\beta,
\end{aligned}
$$

where $\beta=-\gamma \alpha^{2}$ if $\alpha \neq 0$. This means to consider only negative or zero values of $a$ in (83). Indeed, the solutions of (83) for $a>0$ are absent in 14, eqs. (3.1.7)], which are supposed to be the most general solutions of the system (86) and (87). However, the solutions appearing when one considers the solutions of (83) for $a>0$ have their own physical importance. Indeed, Infeld and Hull treat particular cases of their general factorization types $(A)$, $(B)$ and $(E)$ after having made the formal change $\alpha \rightarrow-i \alpha$ [14, pp. 27, 30, $36,46]$.

But the really important point is that in [14], even dealing with their slightly restricted differential equation system (86) and (87), they do not give the general solutions but simply particular ones, since they only consider particular solutions of the Riccati equation with constant coefficients (86). They only consider two such solutions when $\alpha \neq 0$ and another two when $\alpha=0$.

We would like to point out three main aspects now. First, we will treat the differential equation system (83) and (84) for all real values of $a$ and $b$. We will find the general solutions of the system by first considering the general solution of the Riccati equation (83). Second, we will prove that all the solutions included in the classic paper [14 are particular cases of 
that general solutions. Moreover, there is no need of making formal complex changes of parameters for obtaining some of the relevant physical solutions, since they already appear in the general ones. Thirdly, we will see that rather than having four general basic types of factorizable problems $(A),(B),(C)$ and $(D)$, where $(B),(C)$ and $(D)$ could be considered as limiting forms of (A) [14, p. 28], there exist indeed three general basic types of factorizable problems which include the previously mentioned as particular cases, and they are classified by the simple distinction of what sign takes $a$ in (83). The distinction by the sign of $a$ have indeed a deeper geometrical meaning, but we will not go further in this aspect here. See [3], Sec. 4] for more details.

Moreover, the mentioned lack of generality seems to have been propagated to later works trying to generalize the Factorization Method as exposed in [14. See for example some works by Humi [10, 11, 12, 13]. There, more general results could be obtained, in principle, by considering negative values of certain constant appearing in his reasoning and the general solution of the Riccati equation which appears rather than particular ones. For the last two of these references, it would be necessary to consider the general solution of matrix Riccati equations, which may in turn be formulated by means of certain non-linear superposition principle. At this point, it could be of practical use part of the extensive work in the field done by Winternitz and coworkers (see e.g. [24, 25, 21] and references therein).

So, let us find the general solutions of (83) and (84). They are just the same as that of the differential equation system (56) and (57), simply identifying $y(x)$ as $k_{1}(x)$ and $z(x)$ as $k_{0}(x)$, with the same notation for the constants. The results are shown in Table 4 .

Next we show how these solutions reduce to the ones contained in [14. For the case $a<0$, taking $B \rightarrow 0$ we recover the factorization type $(A)$ of Infeld and Hull [14, eq. (3.1.7a)]. And taking $B \rightarrow i$, with a slight generalization of the values $B$ can take, we obtain their type $(B)$ (see eq. $(3.1 .7 b))$. For practical cases of physical interest, they use these factorization types after making the formal change $\alpha \rightarrow-i \alpha$ [14, pp. 27, 30, 36, 46]. The same results would be obtained if one considers the limiting cases $B \rightarrow 0$ or $B \rightarrow 1$, respectively, when $a>0$, so there is no need of making such formal changes. For the case $a=0$, taking $B \rightarrow \infty$ or $B \rightarrow 0$ we recover their factorization types $(C)$ and $(D)$ (see their equations $(3.1 .7 c)$ and $(3.1 .7 d)$ ), respectively. Remember that our convention for the constants appearing in equations (83) and (84) differs slightly from that of equations (3.1.5) of [14], reproduced here as (86) and (87) with Greek characters for the constants. 
We show as well some limiting cases of $B$ which give us the particular solutions used in the construction of the general ones. Remember that the limits $B \rightarrow \infty$ should be taken with care. The arbitrary constant $D$ appearing in the table is not defined exactly in the same way in all its occurrences but it always reflects the fact of having an arbitrary constant wherever it appears.

Let us now try to further generalize (80) to higher powers of $m$. If we try

$$
k(x, m)=k_{0}(x)+m k_{1}(x)+m^{2} k_{2}(x),
$$

substituting it into (79) we obtain

$$
\begin{aligned}
& L(m)-L(m+1)=4 m^{3} k_{2}^{2}+2 m^{2}\left(3 k_{1} k_{2}+3 k_{2}^{2}+k_{2}^{\prime}\right) \\
& +2 m\left(k_{1}^{2}+3 k_{1} k_{2}+2 k_{2}^{2}+2 k_{0} k_{2}+k_{1}^{\prime}+k_{2}^{\prime}\right)+\ldots,
\end{aligned}
$$

where the dots stand for terms not involving $m$. Since the coefficients of powers of $m$ must be constant, from the term in $m^{3}$ we have $k_{2}=$ Const. From the other terms, if $k_{2} \neq 0$ we obtain that both of $k_{1}$ and $k_{0}$ have to be constant as well. That is, a case of the trivial solution $k(x, m)=f(m)$. The same procedure can be used to show that further generalizations to higher powers of $m$ give no new solutions [14].

Let us try now the simplest generalization of (80) to inverse powers of $m$. Assuming $m \neq 0$, we propose

$$
k(x, m)=\frac{k_{-1}(x)}{m}+k_{0}(x)+m k_{1}(x) .
$$

Substituting into (79) we obtain

$$
L(m)-L(m+1)=\frac{(2 m+1) k_{-1}^{2}}{m^{2}(m+1)^{2}}-2 \frac{k_{0} k_{-1}}{m(m+1)}+\frac{(2 m+1) k_{-1}^{\prime}}{m(m+1)}+\ldots,
$$

where the dots denote now the right hand side of (82). Then, in addition to the equations (83) and (84) the following have to be satisfied

$$
k_{-1}^{2}=e, \quad k_{0} k_{-1}=f, \quad k_{-1}^{\prime}=g,
$$

where the right hand side of these equations are constants. Is easy to prove that the only non-trivial new solutions appear when $k_{-1}(x)=q$, with $q$ nonvanishing constant, $k_{0}(x)=0$ and $k_{1}(x)$ is not constant. We have to consider 


\begin{tabular}{|c|c|c|c|}
\hline Sign of $a$ & $k_{1}(x)$ and limits & $k_{0}(x)$ and limits & Comments \\
\hline$a=c^{2}>0$ & $\begin{array}{l}c \frac{B \sinh (c(x-A))-\cosh (c(x-A))}{B \cosh (c(x-A))-\sinh (c(x-A))} \\
\stackrel{B \rightarrow \infty}{\longrightarrow} c \tanh (c(x-A)) \\
\stackrel{B \rightarrow 0}{\longrightarrow} c \operatorname{coth}(c(x-A)) \\
\stackrel{B \rightarrow \mp 1}{\longrightarrow} \pm c\end{array}$ & $\begin{array}{l}\frac{\frac{b}{c}\{B \sinh (c(x-A))-\cosh (c(x-A))\}+D}{B \cosh (c(x-A))-\sinh (c(x-A))} \\
\stackrel{B \rightarrow \infty}{\longrightarrow} \frac{b}{c} \tanh (c(x-A))+\frac{D}{\cosh (c(x-A))} \\
\stackrel{B \rightarrow 0}{\longrightarrow} \frac{b}{c} \operatorname{coth}(c(x-A))+\frac{D}{\sinh (c(x-A))} \\
\stackrel{B \rightarrow \mp 1}{\longrightarrow} \pm \frac{b}{c}+D \exp (\mp c(x-A))\end{array}$ & $\begin{array}{l}\text { See } 69 \\
\text { See text } \\
\text { See } 70\end{array}$ \\
\hline$a=0$ & $\begin{array}{l}\frac{B}{1+B(x-A)} \\
\stackrel{B \rightarrow \infty}{\longrightarrow} \frac{1}{x-A} \\
\stackrel{B \rightarrow 0}{\longrightarrow} 0\end{array}$ & $\begin{array}{l}\frac{b\left(\frac{B}{2}(x-A)^{2}+x-A\right)+D}{1+B(x-A)} \\
\stackrel{B \rightarrow \infty}{\longrightarrow} \frac{b}{2}(x-A)+\frac{D}{x-A} \\
\stackrel{B \rightarrow 0}{\longrightarrow} b(x-A)+D\end{array}$ & $\begin{array}{l}\text { Type }(C) \\
\text { Type }(D)\end{array}$ \\
\hline$a=-c^{2}<0$ & $\begin{array}{l}-c \frac{B \sin (c(x-A))+\cos (c(x-A))}{B \cos (c(x-A))-\sin (c(x-A))} \\
\stackrel{B \rightarrow \infty}{\longrightarrow}-c \tan (c(x-A)) \\
\stackrel{B \rightarrow 0}{\longrightarrow} c \cot (c(x-A)) \\
\stackrel{B \rightarrow \pm i}{\longrightarrow} \pm i c\end{array}$ & $\begin{array}{l}\frac{\frac{b}{c}\{B \sin (c(x-A))+\cos (c(x-A))\}+D}{B \cos (c(x-A))-\sin (c(x-A))} \\
\stackrel{B \rightarrow \infty}{\longrightarrow} \frac{b}{c} \tan (c(x-A))+\frac{D}{\cos (c(x-A))} \\
\stackrel{B \rightarrow 0}{\longrightarrow}-\frac{b}{c} \cot (c(x-A))+\frac{D}{\sin (c(x-A))} \\
\stackrel{B \rightarrow \pm i}{\longrightarrow} \mp i \frac{b}{c}+D \exp (\mp i c(x-A))\end{array}$ & $\begin{array}{l}\text { See } 76 \\
\text { Type }(A) \\
\text { Type }(B)\end{array}$ \\
\hline
\end{tabular}

Table 4: General solutions of the equations (83) and (84), and some limiting cases. $A$ and $B$ are integration constants. The constant $B$ selects the particular solution of (83) in each case. $D$ is not defined always the same way, but always represents an arbitrary constant. 


\begin{tabular}{|c|c|c|c|c|}
\hline Sign of $a$ & $k_{1}(x)$ and limiting cases & $k_{0}(x)$ & $k_{-1}(x)$ & Comments \\
\hline$a=c^{2}>0$ & $\begin{array}{l}c \frac{B \sinh (c(x-A))-\cosh (c(x-A))}{B \cosh (c(x-A))-\sinh (c(x-A))} \\
\stackrel{B \rightarrow 0}{\longrightarrow} c \operatorname{coth}(c(x-A))\end{array}$ & $\begin{array}{l}0 \\
0\end{array}$ & $\begin{array}{l}q \in \mathbb{R} \\
q \in \mathbb{R}\end{array}$ & See text \\
\hline$a=0$ & $\begin{array}{l}\frac{B}{1+B(x-A)} \\
\stackrel{B \rightarrow \infty}{\longrightarrow} \frac{1}{x-A}\end{array}$ & $\begin{array}{l}0 \\
0\end{array}$ & $\begin{array}{l}q \in \mathbb{R} \\
q \in \mathbb{R}\end{array}$ & Type $(F)$ \\
\hline$a=-c^{2}<0$ & $\begin{array}{l}-c \frac{B \sin (c(x-A))+\cos (c(x-A))}{B \cos (c(x-A))-\sin (c(x-A))} \\
\stackrel{B \rightarrow 0}{\longrightarrow} c \cot (c(x-A))\end{array}$ & 0 & $\begin{array}{l}q \in \mathbb{R} \\
q \in \mathbb{R}\end{array}$ & Type $(E)$ \\
\hline
\end{tabular}

Table 5: New solutions of equations (83), (84) and (90). $A$ is an arbitrary constant. $B$ selects the particular solution of (83) for each sign of $a$.

then the general solutions of (83) for each sign of $a$, shown in Table 4 . The new results are shown in Table 5. In this table, to obtain really different new non-trivial solutions, $B$ should be different from \pm 1 in the case $a>0$, and different from 0 in the case $a=0$, otherwise we would obtain constant particular solutions of (83).

For the case $a<0$, taking $B \rightarrow 0$ we recover the factorization type $(E)$ of Infeld and Hull [14, eq. (3.1.7e)]. Again, they use this factorization type for particular cases of physical interest after having made the formal change $\alpha \rightarrow i \alpha$ [14, pp. 46, 47]. The same result is achieved by considering the limiting case $B \rightarrow 0$ in $a>0$. For the case $a=0$, taking $B \rightarrow \infty$ we recover the factorization type $(F)$ (see their equation $(3.1 .7 f)$ ). For all these solutions of (79) of type (89) the expression for $L(m)$ is $L(m)=-a m^{2}-q^{2} / m^{2}+t$, with $t$ an arbitrary real constant, which is also valid for the case $a=0$.

It can be checked that further generalizations of (89) to higher negative powers of $m$ lead to no new solutions apart from the trivial one and that of Tables 1 and 5 .

As a consequence, we have obtained all possible solutions of (79) for 
$k(x, m)$ if it takes the form of a finite sum of terms involving functions of only $x$ times powers of $m$. As a consequence of Corollary 1 we have found six different, and rather general families of Shape-Invariant potentials in the sense of [8] which depend on only one parameter $m$ transformed by translation. These are calculated by means of the formulas (47), (48), (54) and (55). We show the final results in Tables 6, 7 and 8. We would like to remark here several relations that satisfy the functions defined in Table 6 . In the case $a=c^{2}$ we have

$$
f_{+}^{\prime}=c\left(1-f_{+}^{2}\right)=c\left(B^{2}-1\right) h_{+}^{2}, \quad h_{+}^{\prime}=-c f_{+} h_{+},
$$

in the case $a=0$,

$$
f_{0}^{\prime}=-B f_{0}^{2}, \quad h_{0}^{\prime}=-B f_{0} h_{0}+1,
$$

and finally in the case $a=-c^{2}$,

$$
f_{-}^{\prime}=c\left(1+f_{-}^{2}\right)=c\left(B^{2}+1\right) h_{-}^{2}, \quad h_{-}^{\prime}=c f_{-} h_{-},
$$

where the prime means derivative respect to $x$ and the arguments are the same as in the mentioned table, but have been dropped out for simplicity.

\section{Conclusions and outlook}

After a quick review of basic concepts in the theory of factorizable Hamiltonians and Supersymmetric Quantum Mechanics, we have carefully analyzed the equivalence between a generalization of the Factorization Method given in [14] as to allow the relevant parameters to change in an arbitrary way, and the Shape Invariant potentials theory.

We have treated the particularly simple but important case of only one parameter subject to translations, that is, the kind of problems treated by Infeld and Hull in their classic paper. To do that, we have considered the general solutions of certain Riccati equation with constant coefficients rather than particular ones. As a result, we have obtained more general classes of factorizable problems (resp. Shape Invariant partner potentials) than the ones appearing in [14].

On the other hand, the bridge beetween Shape Invariance and factorizable problems has been established more clearly. To this respect, we would like to remark that in the interesting paper $[\mathbf{6}$, Sec. VI] a classification of 


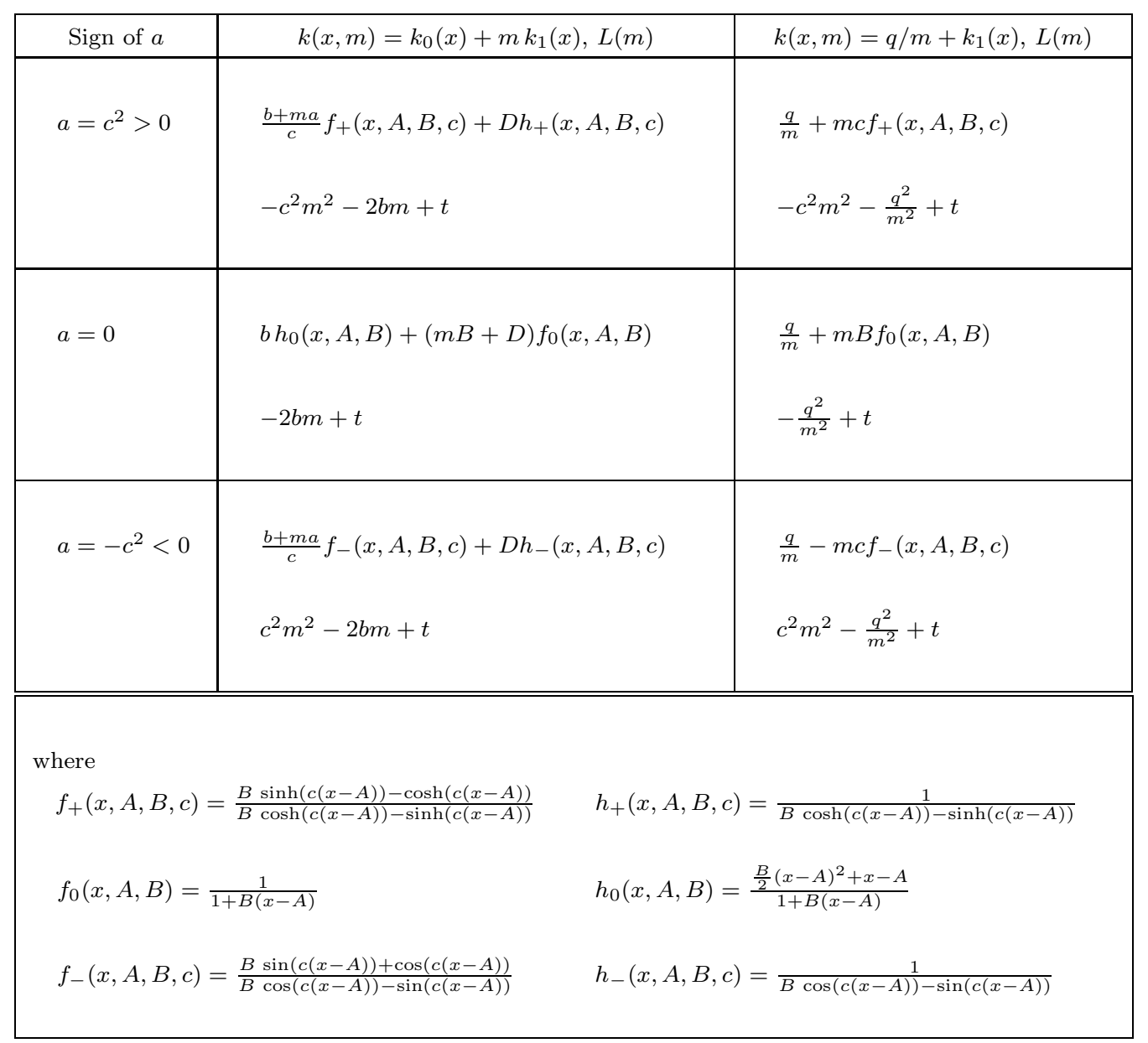

Table 6: General solutions for the two forms of $k(x, m)$ (80) and (89). A, $B, D, q$ and $t$ are arbitrary constants. The constant $B$ selects the particular solution of (83) for each sign of $a$. The constant $b$ is that of (84). 


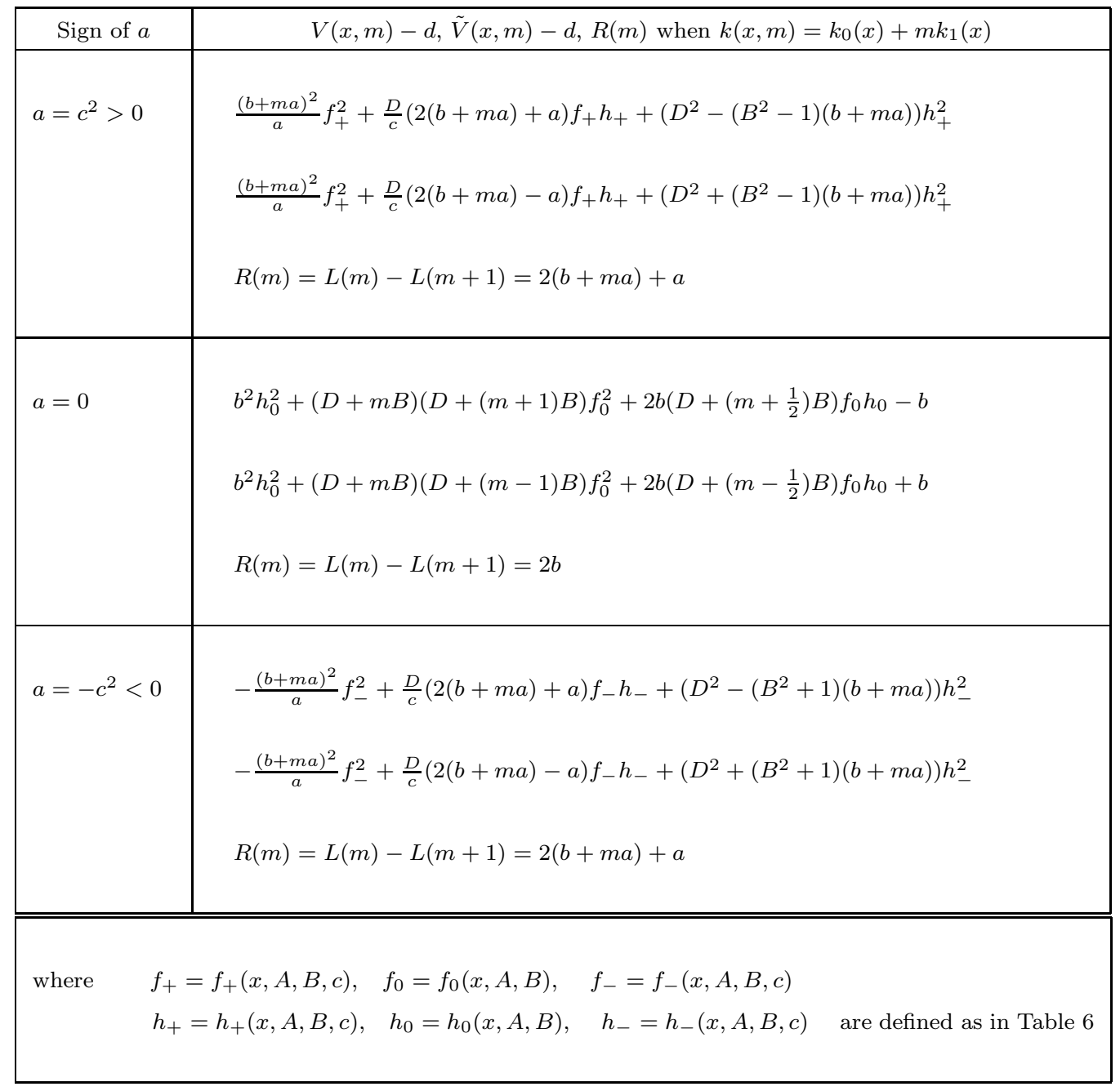

Table 7: Shape-Invariant potentials which depend on one parameter $m$ transformed by traslation, when $k(x, m)$ is of the form (80). A, B, and $D$ are arbitrary constants. The constant $B$ selects the particular solution of (83) for each sign of $a$. The constant $b$ is that of (84). The Shape Invariance condition $\tilde{V}(x, m)=V(x, m-1)+R(m-1)$ is satisfied in all cases. 


\begin{tabular}{|c|c|}
\hline Sign of $a$ & $V(x, m)-d, \tilde{V}(x, m)-d, R(m)$ when $k(x, m)=q / m+m k_{1}(x)$ \\
\hline$a=c^{2}>0$ & $\begin{array}{l}\frac{q^{2}}{m^{2}}+m^{2} c^{2}+2 q c f_{+}-m(m+1) c^{2}\left(B^{2}-1\right) h_{+}^{2} \\
\frac{q^{2}}{m^{2}}+m^{2} c^{2}+2 q c f_{+}-m(m-1) c^{2}\left(B^{2}-1\right) h_{+}^{2} \\
R(m)=L(m)-L(m+1)=\frac{q^{2}}{(m+1)^{2}}-\frac{q^{2}}{m^{2}}+(2 m+1) c^{2}\end{array}$ \\
\hline$a=0$ & $\begin{array}{l}\frac{q^{2}}{m^{2}}+2 q B f_{0}+m(m+1) B^{2} f_{0}^{2} \\
\frac{q^{2}}{m^{2}}+2 q B f_{0}+m(m-1) B^{2} f_{0}^{2} \\
R(m)=L(m)-L(m+1)=\frac{q^{2}}{(m+1)^{2}}-\frac{q^{2}}{m^{2}}\end{array}$ \\
\hline$a=-c^{2}<0$ & $\begin{array}{l}\frac{q^{2}}{m^{2}}-m^{2} c^{2}-2 q c f_{-}+m(m+1) c^{2}\left(B^{2}+1\right) h_{-}^{2} \\
\frac{q^{2}}{m^{2}}-m^{2} c^{2}-2 q c f_{-}+m(m-1) c^{2}\left(B^{2}+1\right) h_{-}^{2} \\
R(m)=L(m)-L(m+1)=\frac{q^{2}}{(m+1)^{2}}-\frac{q^{2}}{m^{2}}-(2 m+1) c^{2}\end{array}$ \\
\hline where & $\begin{array}{lll}f_{+}(x, A, B, c), & f_{0}=f_{0}(x, A, B), \quad f_{-}=f_{-}(x, A, B, c) \\
h_{+}(x, A, B, c), & h_{0}=h_{0}(x, A, B), \quad h_{-}=h_{-}(x, A, B, c) \quad \text { are defined as in Table }\end{array}$ \\
\hline
\end{tabular}

Table 8: Shape-Invariant potentials which depend on one parameter $m$ transformed by traslation, when $k(x, m)$ is of the form (89). $A, B, D$ and $q$ are arbitrary constants. The constant $B$ selects the particular solution of (83) for each sign of $a$. The constant $b$ is that of (84). The Shape Invariance condition $\tilde{V}(x, m)=V(x, m-1)+R(m-1)$ is satisfied in all cases. 
several solutions to the Shape Invariance condition (51) is given. Comparing their ansästze for the superpotential (6.8) with the one proposed by Infeld and Hull, reproduced here as (89), is even more clear the relation between both approaches. In both of them, the solutions can be generalized simply considering the general solutions of a Riccati equation, as we have shown in this article.

But what is even more important is that the use of the properties of the Riccati equation provides a great insight in order to study still unsolved problems as the one suggested in the end of [6, Sec. VI]. That is the subject of another article [4].

Finally, we would like to note, since [14 is a very referenced and used paper, that we have detected one missprint there which may produce later unaccurate results. In the expression of the factorization of general Type B of [14, page 36], $k(x, m)$ should be $d \exp (a x)-a(m+c)$ instead of $d \exp (a x)-$ $m-c$, according to their notation. This missprint is reproduced in their final table of factorizations, page 67. However, the function $r(x, m)$ they give for that $k(x, m)$ is correct.

\section{Acknowledgements.}

One of the authors (A.R.) thanks the Spanish Ministerio de Educación y Cultura for a FPI grant, research project PB96-0717. Support of Spanish DGES (PB96-0717) is also acknowledged.

\section{References}

[1] Cariñena J.F., Marmo G., and Nasarre J., The nonlinear superposition principle and the Wei-Norman method, Int. J. Mod. Phys. 13, 3601-27 (1998).

[2] Cariñena J.F., Marmo G., Perelomov A.M. and Rañada M.F., Related operators and exact solutions of Schrödinger equations, Int. J. Mod. Phys. A 13, 4913-29 (1998).

[3] Cariñena J.F. and Ramos A., Integrability of the Riccati equation from a group theoretical viewpoint, Int. J. Mod. Phys. A 14, 1935-51 (1999). 
[4] Cariñena J.F. and Ramos A., Shape invariant potential depending on $n$ parameters transformed by translation, DFTUZ Preprint 99/09, (1999). Submitted.

[5] Cariñena J.F., Grabowski J. and Ramos A., Reduction of timedependent systems admitting a superposition principle, DFTUZ Preprint 99/07, (1999). Submitted.

[6] Cooper F., Ginocchio J.N. and Khare A., Relationship between supersymmetry and solvable potentials, Phys. Rev. 36 D, 2458-73 (1987).

[7] Davis H.T., Introduction to Nonlinear Differential and Integral Equations, (Dover, New York, 1962).

[8] Gendenshteïn L.É., Derivation of exact spectra of the Schrödinger equation by means of supersymmetry, JETP Lett. 38, 356-9 (1983).

[9] Gendenshteïn L.É. and Krive I.V., Supersymmetry in quantum mechanics, Soviet Phys. Usp. 28, 645-66 (1985).

[10] Humi M., Extension of the Factorization Method, J. Math. Phys. 9, 1258-65 (1968).

[11] Humi M., New types of factorizable equations, Proc. Camb. Phil. Soc. 68, 439-46 (1970).

[12] Humi M., Factorization of systems of differential equations, J. Math. Phys. 27, 76-81 (1986).

[13] Humi M., Novel types of factorisable systems of differential equations, J. Phys. A: Math. Gen. 20, 1323-31 (1987).

[14] Infeld L. and Hull T.E., The Factorization Method, Rev. Mod. Phys. 23, 21-68 (1951).

[15] Lie S. and Scheffers G., Vorlesungen über continuierlichen Gruppen mit geometrischen und anderen Anwendungen, (Teubner, Leipzig, 1893).

[16] Montemayor R. and Salem L.D., Supersymmetry shape invariance and solubility in quantum mechanics, Phys. Rev. A 40, 2170-2173 (1987). 
[17] Murphy G.M., Ordinary Differential equations and their solutions, (Van Nostrand, New York, 1960).

[18] Schrödinger E., A method of determining quantum-mechanical eigenvalues and eigenfunctions, Proc. Roy. Irish Acad. A XLVI, 9-16 (1940).

[19] Schrödinger E., Further studies on solving eigenvalue problems by factorization, Proc. Roy. Irish Acad. A XLVI, 183-206 (1941).

[20] Schrödinger E., The factorization of the hypergeometric equation, Proc. Roy. Irish Acad. A XLVII, 53-54 (1941).

[21] Shnider S. and Winternitz P., Classification of Systems of ordinary differential equations with superposition principles, J. Math. Phys. 25, 3155-65 (1984).

[22] Stahlhofen A., The Riccati equation as a common basis for Supersymmetric Quantum Mechanics and the Factorization Method, Preprint Duke University (1988).

[23] Stahlhofen A., Remarks on the equivalence between the Shape-Invariance condition and the factorisation condition, J. Phys. A: Math. Gen. 22, 1053-8 (1989).

[24] Winternitz P., Lie groups and solutions of nonlinear differential equations, in Nonlinear Phenomena, K.B. Wolf Ed., Lecture Notes in Physics 189, (Springer-Verlag, N.Y., 1983).

[25] Winternitz P., Comments on superposition rules for nonlinear coupled first order differential equations, J. Math. Phys. 25, 2149-50 (1984).

[26] Witten E., Dynamical breaking of Supersymmetry, Nucl. Phys. B 188, 513-54 (1981). 